\title{
Conversión de artrodesis a prótesis total de cadera en México. Reporte de un caso
}

\author{
Martínez-Ruiz FJ,* Montero-García JG, ${ }^{* *}$ Rivera-Reyes C*** \\ Hospital de la Beneficiencia Española de Puebla
}

RESUMEN. Desde 1900, la artrodesis de cadera se consideraba como un tratamiento definitivo, siendo el método de elección para la osteoartritis (OA) de cadera en pacientes jóvenes y otros padecimientos como infecciones o secuelas de enfermedades infantiles, aunque hoy día se ha migrado al uso de prótesis con implantes no cementados. Actualmente, el manejo ortopédico de la osteoartritis en la cadera del paciente joven ha cambiado de forma impresionante, en parte derivado de las consecuencias de la artrodesis a largo plazo en las articulaciones vecinas, por ello presentamos este caso en el que se realiza la conversión a una artroplastía total de cadera (ATC).

Palabras clave: Artrodesis de cadera, artroplastía total de cadera, conversión.

\section{Introducción}

En la actualidad, el manejo ortopédico de la osteoartritis (OA) en la cadera del paciente joven ha cambiado de manera impresionante debido a la alta tasa de falla temprana asociada con artroplastía total cementada. ${ }^{1}$ Desde 1900, la artrodesis de cadera se consideraba como un tratamiento definitivo, ${ }^{2}$ siendo el método de elección para esta enfermedad y otros

\footnotetext{
* Residente del Curso Alta Especialidad en Cirugía Articular.

** Médico adscrito al Servicio de Ortopedia, Titular del Curso de Alta Especialidad en Cirugía Articular.

*** Médico adscrito al Servicio de Ortopedia, Adjunto del Curso de Alta Especialidad en Cirugía Articular, Clínica de Cadera.

Dirección para correspondencia:

Dr. Cristian Rivera-Reyes

Departamento de Ortopedia, Clínica de Cadera, Hospital Beneficencia Española de Puebla

19 Norte Núm. 1001,

Col. Jesús García, C.P. 72090, Puebla, México.

E-mail: cristian_rivera_@hotmail.com
}

Este artículo puede ser consultado en versión completa en http:// www.medigraphic.com/actaortopedica
ABSTRACT. Since 1900, hip fusion was considered a definitive treatment, being the method of choice for osteoarthritis (OA) of the hip in young patients, and others as infections or sequelae of childhood illnesses, although today it has been migrated to the use of prostheses with non-cemented implants. Currently orthopedic management of osteoarthritis in the hip of the young patient has changed dramatically in part derived from the consequences of long-term arthrodesis in adjacent joints, so we present this case where we perform the conversion to total hip arthroplasty.

Key words: Hip arthrodesis, total hip arthroplasty, conversion.

padecimientos como infecciones o secuelas de enfermedades infantiles, ${ }^{2,3}$ aunque hoy en día se ha migrado al uso de implantes no cementados, se siguen presentando tasas elevadas de aflojamiento aséptico como consecuencia de mayor actividad física y demanda funcional, con tasas de revisión de fallas mecánicas casi de 50\%. ${ }^{1}$ Se ha considerado que por las consecuencias a largo plazo de la artrodesis de cadera en las articulaciones vecinas a menudo se recomienda la conversión a una artroplastía total de cadera (ATC), principalmente osteoartritis de la columna vertebral $(60 \%)$, rodilla ipsilateral $(30-75 \%)$, rodilla contralateral $(15-30 \%))^{2,3,4,5}$

Con el deterioro y la incapacidad física la ATC puede estar indicada, ${ }^{4}$ aunque la conversión de una cadera fusionada quirúrgicamente a una ATC es un procedimiento cada vez más raro y muchas veces desafiante. El asesoramiento de los pacientes sobre los resultados esperados es difícil, debido a sus altas expectativas y la rareza del procedimiento. ${ }^{5}$ Como resultado de la conversión puede presentarse claudicación, secundaria a la insuficiencia de musculatura abductora, que incluso puede persistir o ser permanente. ${ }^{3,5}$ Es por ello que el cirujano ortopedista debe dominar múltiples técnicas antes de realizar un procedimiento tan complejo; asimismo, debe conocer los diferentes implantes con los que se llevó a 
cabo la artrodesis de cadera como las placas, tornillos, entre otros. ${ }^{6,7,8}$ En un paciente que ha pasado varias décadas fusionado, este procedimiento puede aliviar el dolor en las articulaciones adyacentes y mejorar el funcionamiento bá$\operatorname{sico}^{7,8,9,10}$

\section{Presentación del caso}

Masculino de 61 años de edad, campesino, antecedente de fractura de cadera derecha de 20 años de evolución, se complicó con necrosis femoral proximal, tratado en 1994 con artrodesis de cadera con placa tipo Cobra (Figura 1), evolucionando con coxalgia derecha y dificultad para actividades de la vida cotidiana, acortamiento de miembro pélvico de $4 \mathrm{~cm}$, con un resultado de Harris de 43, con marcha Trendelenburg, uso de apoyos técnicos, con arcos de movilidad limitados. Se tomaron radiografías y tomografía axial computarizada (TAC) simple de pelvis, en las que se observó defecto acetabular Paprosky I. Se realizó cirugía el 06 de Septiembre de 2016 con conversión de artrodesis de cadera derecha a prótesis total primaria con abordaje lateral directo, retiro de material de osteosíntesis, vástago cilíndrico, con recubrimiento poroso extenso de apoyo diafisario

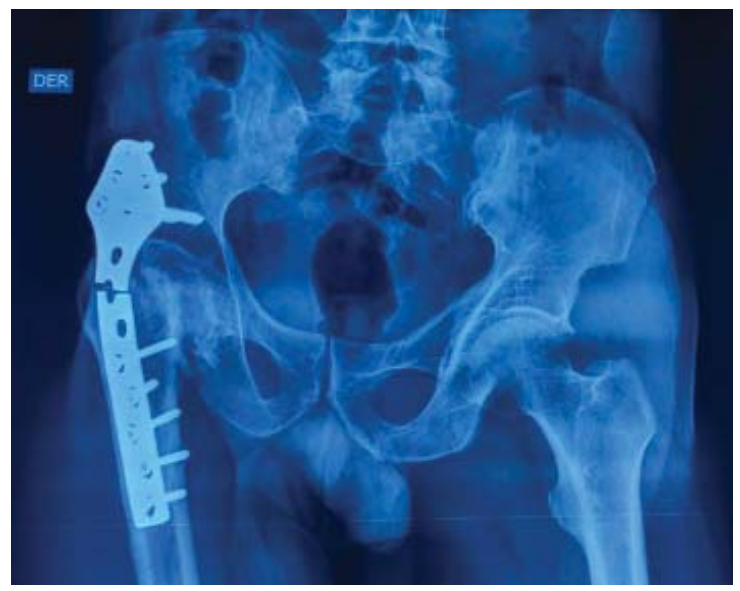

Figura 1: Radiografías preoperatorias.
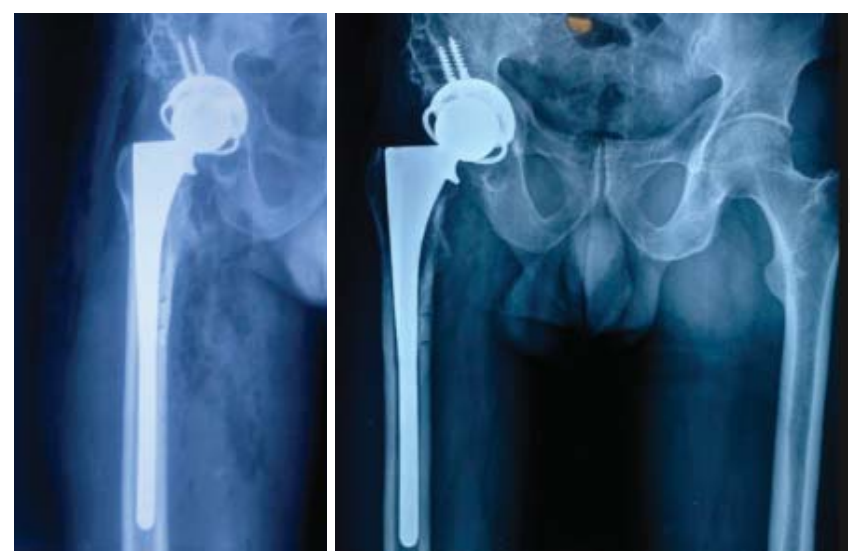

Figura 2: Radiografías postoperatorias inmediatas. no cementado $\left(\right.$ Reach, Biomet $\left.{ }^{\circledR}\right)$, con copa primaria no cementada (Mallory Head, Biomet ${ }^{\circledR}$ ), liner constreñido (Free$\operatorname{dom}^{\circledR}$ ) y cabeza de $36 \mathrm{~mm}$ (Figura 2).

Una vez realizado el procedimiento quirúrgico se evaluó el resultado funcional con la escala de Harris, en el período postoperatorio haciendo cortes con el tiempo a uno, seis y 12 meses (Figura 3).

\section{Discusión}

La artroplastía total de cadera para caderas fusionadas se realizó en 1970 y posteriormente se ha reportado una reconstrucción quirúrgica exitosa de artrodesis de cadera con artroplastía total de articulaciones. La ATC ayudó a estos pacientes a mejorar las actividades de la vida diaria y sentían una satisfacción general. La cadera fusionada secundaria a enfermedades, como artritis piogénica o traumatismo infantil, puede causar contractura adyacente de los tejidos blandos y atrofia haciendo que el abordaje quirúrgico sea muy difícil debido a extensas cicatrices, tejido fibroso y distorsión de los hitos anatómicos. ${ }^{11}$

Kyung-Soon et al. ${ }^{11}$ realizaron la conversión a ATC en 25 pacientes con cadera fusionada, demostrando que los implantes no cementados tipo cerámica en cerámica pueden proporcionar buenos resultados clínicos tempranos. Asimismo, Rutz et al. ${ }^{12}$ mostraron que la ATC después de la anquilosis espontánea dio mejores resultados funcionales en comparación con ATC después de la fusión quirúrgica (artrodesis cadera). Consideramos que estos resultados se debieron a un mejor entendimiento de la musculatura abductora, ya que los pacientes con artrodesis de cadera a menudo presentaban deficiencia del aparato abductor de la cadera derivado de los implantes y abordaje quirúrgico.

Jain et al. ${ }^{1}$ publicaron su revisión sistemática realizada en la Universidad de Leeds del Reino Unido en la que compararon los resultados funcionales y de satisfacción en pacientes sometidos a una artrodesis de cadera con una subsecuente conversión a ATC, con un total de ocho estudios con 249 caderas con artrodesis y 11 estudios que incluían 579
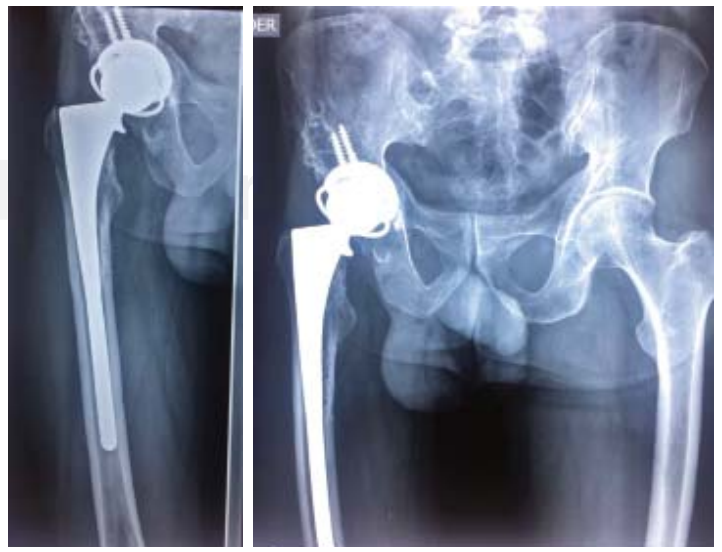

Figura 3: Radiografías postoperatorias a los 12 meses. 
pacientes con una conversión a ATC, revelando resultados inconsistentes con respecto al alivio del dolor. Asimismo, reportaron complicaciones en hasta $54 \%$, más comúnmente debido a fallo mecánico, infección profunda y parálisis nerviosa. Mientras que la artrodesis de cadera puede proporcionar alivio del dolor y la satisfacción del paciente, la artroplastía de conversión se asocia con una tasa de complicación inaceptablemente alta. Sin embargo, considerando nuestro paciente sometido a conversión a ATC, los resultados funcionales fueron buenos y excelentes al primer mes, a los seis y 12 meses con una escala de cadera de Harris de 72, 92 y 96, respectivamente. Consideramos que mucho tiene que ver lo corto del período de evaluación.

En el entendimiento de que es un procedimiento complejo, en nuestro paciente se empleó un liner constreñido $\left(\right.$ Freedom ${ }^{\circledR}$ ), siendo factor importante en el manejo del aparato abductor deficiente.

Villanueva et al. reportaron resultados interesantes de 21 casos en 20 pacientes con cadera fusionada, en 13 caderas se había realizado procedimiento quirúrgico y en ocho o más se debió a una fusión no quirúrgica con un seguimiento mínimo de tres años, el promedio de edad fue 58.5 años, demostrando que aunque la conversión de artrodesis a ATC es un procedimiento desafiante, pero acertado según el resultado clínico a medio plazo observado, sugieren que los factores pronósticos tales como el tipo de fusión (extra versus intraarticular), la edad y el tiempo de conversión, el número de operaciones anteriores, la duración de la fusión, el diagnóstico que conduce a la fusión de cadera, la edad de fusión y el sexo no se asociaron con el resultado clínico tras la conversión a ATC y por otro lado, deben utilizarse con precaución al establecer indicaciones y expectativas postquirúrgicas. Considerando estas aportaciones en nuestro paciente, un masculino de 61 años de edad con una artrodesis por secuela de fractura en cadera de 23 años de evolución y una placa Cobra como implante, se realizó una evaluación de este último que aportara características anatómicas suficientes acerca de la reserva ósea y calidad de la musculatura en la cadera y siendo objetivos con las expectativas del paciente y sus familiares, es decir, la artrodesis de cadera con placa Cobra es un procedimiento que compromete el trocánter mayor e inserciones musculares, alterando el momento abductor, por lo que se decidió la colocación del liner constreñido y vástago de apoyo diafisario, con lo que se evita la posibilidad de luxación y la presencia de fractura periprotésica debido a los orificios diafisarios por el implante previo.

Al final se consideró que la conversión de artrodesis a artroplastía total de cadera es un procedimiento complejo que requiere de una planeación preoperatoria cuidadosa con una correcta selección del implante, tomando en cuenta las características anatómicas de cada caso.

Conflicto de intereses: Los autores y los centros de investigación con los que están afiliados no han recibido pagos financieros u otros beneficios de cualquier entidad comercial relacionada con el tema de este artículo.

Bibliografía

1. Jain S, Giannoudis PV. Arthrodesis of the hip and conversion to total hip arthroplasty. A systematic review. J Arthroplasty. 2013; 28(9): 1596-602.

2. Villanueva M, Sobrón FB, Parra J, Rojo JM, Chana F, Vaquero J. Conversion of arthrodesis to total hip arthroplasty: clinical outcome, complications, and prognostic factors of 21 consecutive cases. HSS J. 2013; 9(2): 138-44.

3. Joshi AB, Markovic L, Hardinge K, Murphy JC. Conversion of a fused hipto total hip arthroplasty. J Bone Joint Surg Am. 2002; 84-A(8): 1335-41.

4. Reikerås $\mathrm{O}$, Bjerkreim I, Gundersson R. Total hip arthroplasty for arthrodesed hips: 5-to 13-year results. J Arthroplasty. 1995; 10(4): 529-31.

5. Richards CJ, Duncan CP. Conversion of hip arthrodesis to total hip arthroplasty: survivorship and clinical outcome. J Arthroplasty. 2011; 26(3): 409-13.

6. Panagiotopoulos KP, Robbins GM, Masri BA, Duncan CP. Conversion of hip arthrodesis to total hip arthroplasty. Instr Course Lect. 2000; 50: 297-305.

7. Whitehouse MR, Duncan CP. Conversion of hip fusion to total hip replacement. Bone Joint J. 2013; 95-B(11 Supple A): 114-9.

8. Aderinto J, Lulu OB, Backstein DJ, Safir O, Gross AE. Functional results and complications following conversion of hip fusion to total hip replacement. J Bone \& Joint Surg Br. 2012; 94(11 Supple A). 3641.

9. Beaulé PE, Matta JM, Mast JW. Hip arthrodesis: current indications and techniques. J Am Acad Orthop Surg. 2002; 10(4): 249-58.

10. Drexler M, Reischl N, Dwyer T, Ng DJ, Attar FG, Cameron HU. Hip conversion: don't promise too much. Seminars in Arthroplasty. 2012; 23(3): 176-8.

11. Park KS, Yoon TR, Lee TM, Ahn YS. Ceramic on ceramic hip arthroplasty in fused hips. Indian J Orthop. 2015; 49(3): 336-41.

12. Rutz E, Schäfer D, Valderrabano V. Total hip arthroplasty after hip joint ankylosis. J Orthop Sci. 2009; 14(16): 727-31. 